\title{
Coherent control of optical bistability in an open $\Lambda$-type three-level atomic system
}

\author{
Zhen Wang, ${ }^{1}$ Ai-Xi Chen, ${ }^{2}$ Yanfeng Bai, ${ }^{1}$ Wen-Xing Yang, ${ }^{1,3, *}$ and Ray-Kuang Lee ${ }^{3}$ \\ ${ }^{1}$ Department of Physics, Southeast University, Nanjing 210096, China \\ ${ }^{2}$ Department of Applied Physics, School of Basic Science, East China Jiaotong University, Nanchang 330013, China \\ ${ }^{3}$ Institute of Photonics Technologies, National Tsing-Hua University, Hsinchu 300, Taiwan \\ ${ }^{*}$ Corresponding author: wenxingyang@seu.edu.cn
}

Received June 13, 2012; revised August 17, 2012; accepted August 27, 2012;

posted August 28, 2012 (Doc. ID 170277); published September 24, 2012

\begin{abstract}
We analyze the hybrid absorptive-dispersive optical bistability (OB) behavior in an open $\Lambda$-type three-level atomic system by using a microwave field to drive the hyperfine transition between two lower states, along with the consideration of incoherent pumping and spontaneously generated coherence. Different from the closed system, we show that the bistable threshold intensity and related hysteresis loop can be controlled by adjusting the ratio between atomic injection and exit rates. More interestingly, the appearance and disappearance of $\mathrm{OB}$ can be transformed mutually by varying the relative phase of three coherent fields under the condition of a strong spontaneously generated coherence. The manipulation of OB behavior through the intensity of the microwave field and the atomic cooperation parameter is also analyzed. (c) 2012 Optical Society of America
\end{abstract}

OCIS codes: $\quad 160.4330,190.1450$.

\section{INTRODUCTION}

Over the past few decades, there has been significant research on quantum optical phenomena with atomic coherence and quantum interference [1,2], such as coherent population trapping and electromagnetically induced transparency (EIT) [3] , amplification and lasing without population inversion [프, enhancing Kerr nonlinearity [5], multiwave mixing [ㅇ-으, optical solitons [9-13], etc. Meanwhile, there is also a great deal of interest in the phenomenon of optical bistability (OB) [14-21], in view of its potential application for optoelectronic devices [22]. Besides atomic systems, OB happens in other kinds of systems, such as semiconductor structures that include quantum wells in an optical ring cavity [14-21]. These studies have shown that field-induced transparency and quantum interference effects could significantly decrease the OB threshold intensity. The effects of the phase fluctuations and squeezed state fields on the $\mathrm{OB}$ have subsequently been studied [23-25].

However, almost all of these studies are considered with a closed system. The closed three-level $\Lambda$-type atomic system is one of the most important systems used in the studies mentioned above. In this regard, OB in a closed three-level $\Lambda$-type atomic system inside an optical cavity has been observed experimentally $[2,16]$. Furthermore, some researchers have shown that squeezed state field [23-25], spontaneously generated coherence (SGC) [26], atomic cooperation parameter, and the intensity of microwave field play a crucial role in controlling the bistable threshold intensity and related hysteresis loop structure [14-26].

Based on the study of OB behavior in a closed three-level atomic system $[\underline{2}, \underline{14}-\underline{16}]$, in this work, we propose a new scheme in an open $\Lambda$-type three-level atomic system to investigate $\mathrm{OB}$, which is driven by a resonant microwave field with incoherent pumping and SGC. The present scheme is drastically different from the schemes for controlling the OB behavior in a closed system [2,14-16]. First, we show that the OB threshold intensity in the open system is smaller than that in the corresponding closed system. A smaller threshold intensity is obviously favorable from the viewpoint of practical applications. Second, we demonstrate that the behavior of OB including threshold intensity and the hysteresis loop can be controlled by varying the ratio between atomic injection and exit rates. Third, we find that the behavior of $\mathrm{OB}$ is very sensitive to the relative phase of three coherent fields even with the existence of a small intensity of SGC. More interestingly, we also find that the appearance and disappearance of $\mathrm{OB}$ can be transformed mutually by adjusting the relative phase of three coherent fields when the SGC is strong enough. If the microwave field is not used, except for the case with a nonzero frequency detuning $\left(\Delta_{p}\right.$ or $\left.\Delta_{c}\right)$ [16], there is no OB behavior to observe.

\section{MODEL AND MOTION EQUATIONS}

Let us consider an open three-level $\Lambda$-type atomic system as shown in Fig. 1. The transition frequency, $\omega_{13}$ for $|1\rangle \leftrightarrow|3\rangle$, is driven by a weak probe field $E_{p}$ with angular frequency $\omega_{p}$ and Rabi frequency $2 \Omega_{p}$. The transition frequency, $\omega_{23}$ for $|2\rangle \leftrightarrow|3\rangle$, is driven by a coherent coupled field $E_{c}$ with angular frequency $\omega_{c}$ and Rabi frequency $2 \Omega_{c}$. A resonant microwave field $E_{d}$ with angular frequency $\omega_{d}$ and Lamor frequency $\Omega_{d}$ is used to couple Zeeman sublevels $|1\rangle$ and $|2\rangle$ through an allowed magnetic dipole transition. An incoherent pump field with the pumping rate $\Lambda$ is applied between levels $|1\rangle \leftrightarrow|3\rangle$. $J_{1}$ and $J_{2}$ are the atomic injection rates for level $|1\rangle$ and $|2\rangle$, respectively. $r_{0}$ is the atomic exit rate. We also assume that the number of interacting atomic is constant, so $J_{1}+J_{2}=r_{0}$. If we take the level $|1\rangle$ as the energy ground and choose $H_{0}=\left(\omega_{p}-\omega_{c}\right)|2\rangle\left\langle 2\left|+\omega_{p}\right| 3\right\rangle|3\rangle$ in the interaction 


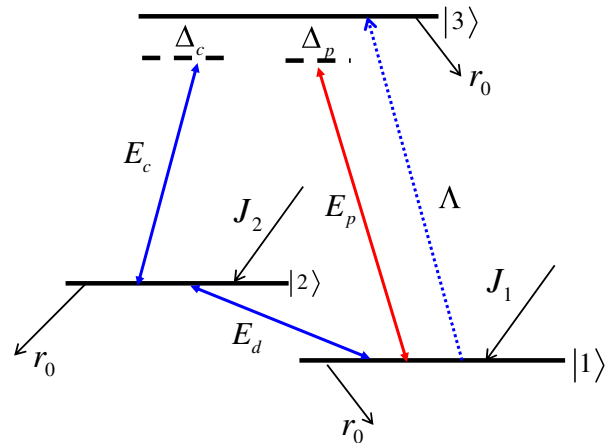

Fig. 1. (Color online) Schematic diagram of an open three-level $\Lambda$-type atomic system.

picture and under the rotating-wave approximation, the interaction Hamiltonian of this system is given as follows $(\hbar=1)$ :

$$
\begin{aligned}
H_{\mathrm{int}}= & \left(\Delta_{p}-\Delta_{c}\right)|2\rangle\left\langle 2\left|+\Delta_{p}\right| 3\right\rangle\langle 3| \\
& -\left(\Omega_{d}|2\rangle\left\langle 1\left|+\Omega_{p}\right| 3\right\rangle\left\langle 1\left|+\Omega_{c}\right| 3\right\rangle\langle 2|+\text { H.c. }\right),
\end{aligned}
$$

where the symbol H.c. means the Hermitian conjugate. $\Delta_{p}=$ $\omega_{31}-\omega_{p}$ and $\Delta_{c}=\omega_{32}-\omega_{c}$ are the detunings of the probe field and the coherent coupling field, respectively. $\Omega_{n}(n=p, c, d)$ presents one-half Rabi and Lamor frequencies for the respective transitions, i.e., $\Omega_{p}=\mu_{31} E_{p} / 2 \hbar, \Omega_{c}=$ $\mu_{32} E_{c} / 2 \hbar$ and $\Omega_{d}=\mu_{12} E_{d} / 2 \hbar$, where $\mu_{i j}$ denotes the dipole moment for the transition between levels $|i\rangle$ and $|j\rangle$.

With the density-matrix formalism, we study the dynamic in our proposed scheme. By adopting the standard approaches [27], the density-matrix equations can be given as follows:

$$
\begin{aligned}
& \dot{\rho}_{11}=i \Omega_{d}^{*} \rho_{21}-i \Omega_{d} \rho_{12}+i \Omega_{p}^{*} \rho_{31}-i \Omega_{p} \rho_{13}-\Lambda \rho_{11}+\gamma_{21} \rho_{22} \\
& +\gamma_{31} \rho_{33}+J_{1}-r_{0} \rho_{11} \text {, } \\
& \dot{\rho}_{22}=i \Omega_{d} \rho_{12}-i \Omega_{d}^{*} \rho_{21}+i \Omega_{c}^{*} \rho_{32}-i \Omega_{c} \rho_{23}-\gamma_{21} \rho_{22} \\
& +\gamma_{32} \rho_{33}+J_{2}-r_{0} \rho_{22} \text {, } \\
& \dot{\rho}_{33}=i \Omega_{c} \rho_{23}-i \Omega_{c}^{*} \rho_{32}+i \Omega_{p} \rho_{13}-i \Omega_{p}^{*} \rho_{31}+\Lambda \rho_{11} \\
& -\left(\gamma_{31}+\gamma_{32}\right) \rho_{33}-r_{0} \rho_{33} \text {, } \\
& \dot{\rho}_{12}=i \Omega_{d}^{*}\left(\rho_{22}-\rho_{11}\right)+\left[i\left(\Delta_{p}-\Delta_{c}\right)-\frac{\gamma_{21}}{2}\right] \rho_{12} \\
& +i \Omega_{p}^{*} \rho_{32}-i \Omega_{c} \rho_{13}+\sqrt{\gamma_{31} \gamma_{32}} p \eta \rho_{33}, \\
& \dot{\rho}_{13}=i \Omega_{p}^{*}\left(\rho_{33}-\rho_{11}\right)+i \Omega_{d}^{*} \rho_{23}-i \Omega_{c}^{*} \rho_{12} \\
& +\left[i \Delta_{p}-\frac{1}{2}\left(\gamma_{32}+\gamma_{31}\right)\right] \rho_{13}, \\
& \dot{\rho}_{23}=i \Omega_{d} \rho_{13}-i \Omega_{p}^{*} \rho_{21}+i \Omega_{c}^{*}\left(\rho_{33}-\rho_{22}\right) \\
& +\left[i \Delta_{c}-\frac{1}{2}\left(\gamma_{21}+\gamma_{31}+\gamma_{32}\right)\right] \rho_{23},
\end{aligned}
$$

together with $\rho_{i j}=\rho_{j i}^{*}$ and $\sum_{j=1}^{3} \rho_{j j}=1$. Here, the overdots denote the derivation with respect to time $t$. The population decay rates are added phenomenologically in the above density matrix equations, where the population decay between level $|i\rangle$ and $|j\rangle$ is denoted by $\gamma_{i j}$. The term $p \sqrt{\gamma_{31} \gamma_{32}} \eta \rho_{33}$ represents the SGC effect resulting from cross coupling emission $|3\rangle \leftrightarrow|1\rangle$ and $|3\rangle \leftrightarrow|2\rangle$, where $p=\cos \theta=\mu_{31} \cdot \mu_{32} /\left|\mu_{31}\right|\left|\mu_{32}\right|$ with $\theta$ the angle between the two induced dipole moments $\mu_{31}$ and $\mu_{32}$ [14]. It is obvious that the parameter $\eta$ presents the strength of SGC effect and varies with the value of $\theta$. $\eta=1$ presents the largest strength of the SGC, while $\eta=0$ presents no SGC effect. When $J_{1}=J_{2}=r_{0}=0$, the set of equations in (2)-(7) can be reduced case of a closed $\Lambda$-type three-level atomic system [28].

The properties of a open system depends not only on amplitudes but also on the phases of probe and control fields due to the SGC effect. In this case, we have to treat the Rabi frequencies as complex parameters [29]. We define $\phi_{p}, \phi_{c}$, and $\phi_{d}$ as the phases of the probe, control, and microwave fields, respectively. Then, we have $\Omega_{p}=G_{p} e^{-i \phi_{p}}, \Omega_{c}=G_{c} e^{-i \phi_{c}}$, and $\Omega_{d}=\Omega_{d}^{*}=G_{d}$ with $G_{p}, G_{c}$, and $G_{d}$ are real parameters. Other density elements defined as $\sigma_{i i}=\rho_{i i}(i=1,2,3)$, $\sigma_{31}=\rho_{31} e^{i \phi_{p}}, \sigma_{32}=\rho_{32} e^{i \phi_{c}}$ and $\sigma_{21}=\rho_{21} e^{i\left(\phi_{p}-\phi_{c}\right)}$. In this way, the set of equations in (2)-(7) can be rewritten as

$$
\begin{aligned}
& \dot{\sigma}_{11}=i G_{d}\left(\sigma_{21} e^{-i \phi}-\sigma_{12} e^{i \phi}\right)+i G_{p}\left(\sigma_{31}-\sigma_{13}\right) \\
& +\gamma_{21} \sigma_{22}+\gamma_{31} \sigma_{33}+J_{1}-r_{0} \sigma_{11} \text {, } \\
& \dot{\sigma}_{22}=i G_{d}\left(\sigma_{12} e^{i \phi}-\sigma_{21} e^{-i \phi}\right)+i G_{c}\left(\sigma_{32}-\sigma_{23}\right)-\gamma_{21} \sigma_{22} \\
& +\gamma_{32} \sigma_{33}+J_{2}-r_{0} \sigma_{22} \text {, } \\
& \dot{\sigma}_{33}=i G_{c}\left(\sigma_{23}-\sigma_{32}\right)+i G_{p}\left(\sigma_{13}-\sigma_{31}\right) \\
& +\Lambda \sigma_{11}-\left(\gamma_{31}+\gamma_{32}\right) \sigma_{33}-r_{0} \sigma_{33} \text {, } \\
& \dot{\sigma}_{12}=i G_{d} e^{-i \phi}\left(\sigma_{22}-\sigma_{11}\right)+i\left[\left(\Delta_{p}-\Delta_{c}\right)-\frac{\gamma_{21}}{2}\right] \sigma_{12} \\
& +i G_{p} \sigma_{32}-i G_{c} \sigma_{13}+\sqrt{\gamma_{31} \gamma_{32}} p \eta e^{-i \phi} \sigma_{33} \text {, } \\
& \dot{\sigma}_{13}=i G_{p}\left(\sigma_{33}-\sigma_{11}\right)+i G_{d} \sigma_{23} e^{-i \phi}-i G_{c} \sigma_{12} \\
& +\left[i \Delta_{p}-\frac{1}{2}\left(\gamma_{32}+\gamma_{31}\right)\right] \sigma_{13} \text {, } \\
& \dot{\sigma}_{23}=i G_{d} \sigma_{13} e^{i \phi}-i G_{p} \sigma_{21}+i G_{c}\left(\sigma_{33}-\sigma_{22}\right) \\
& +\left[i \Delta_{c}-\frac{1}{2}\left(\gamma_{21}+\gamma_{31}+\gamma_{32}\right)\right] \sigma_{23},
\end{aligned}
$$

where $\phi=\phi_{p}-\phi_{c}$ is the relative phase difference of the probe field and the driving fields. Similarly, Eq. (8) are restricted by $\sigma_{i j}=\sigma_{j i}^{*}$ and $\sigma_{11}+\sigma_{22}+\sigma_{33}=1$.

Now, we consider a medium of length $L$ composed by the atomic ensemble described above, which is immersed in a unidirectional ring cavity as shown in Fig. 2. For simplicity, we assume that both mirrors 3 and 4 are $100 \%$ in the reflectivity, 


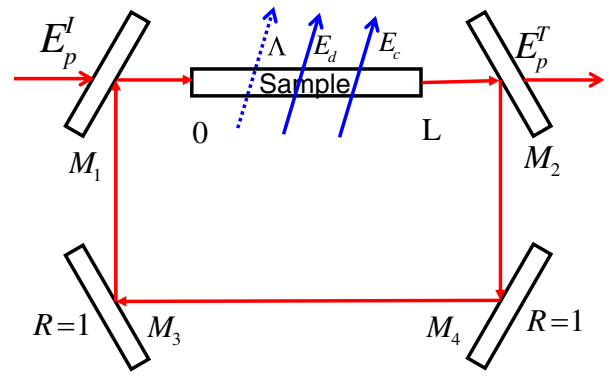

Fig. 2. (Color online) Schematic setup of a unidirectional ring cavity containing an atomic sample with the length $L . E_{p}^{I}$ and $E_{p}^{T}$ are the incident and transmitted fields, respectively. $E_{c}, E_{d}$, and $\Lambda$ represent the coupling field, microwave driving field, and incoherent pumping, that are not circulating inside the cavity.

and the reflection and transmission coefficient for mirrors 1 and 2 are denoted by $R$ and $T$ (with $R+T=1$ ), respectively. The total electromagnetic field can be written as $E=E_{p} e^{-i \omega_{p} t}+E_{c} e^{-i \omega_{c} t}+E_{d} e^{-i \omega_{d} t}+$ c.c., where only the probe field $E_{p}$ circulates in the ring cavity. The propagation of probe field in the medium is governed by Maxwell equation, then, under the slowly varying envelope approximation [27], the dynamic equation for the probe field is

$$
\frac{\partial E_{p}}{\partial t}+c \frac{\partial E_{p}}{\partial z}=i \frac{\omega_{p}}{2 \varepsilon_{0}} P\left(\omega_{p}\right)
$$

where $P\left(\omega_{p}\right)=N \mu_{13} \rho_{31}$ is the slowly oscillating term for the induce polarization in the transition $|3\rangle \leftrightarrow|1\rangle$, with the electron density in the sample denoted by $N$. In Eqs. (8)-(13), the parameters $\varepsilon_{0}$ and $c$ are the permittivity of free space and the speed of light in vacuum, respectively. In the steady-state case, the time derivative in Eq. (14) $\partial E_{p} / \partial t$ is set to zero; thus, we can obtain the amplitude of the probe field as follows:

$$
\frac{\partial E_{p}}{\partial z}=i \frac{\omega_{p}}{2 c \varepsilon_{0}} P\left(\omega_{p}\right)
$$

The input probe field $E_{p}^{I}$, entering the cavity under the assumption for a perfectly tuned ring cavity, satisfies the following boundary conditions together with the transmitted field $E_{p}^{T}$

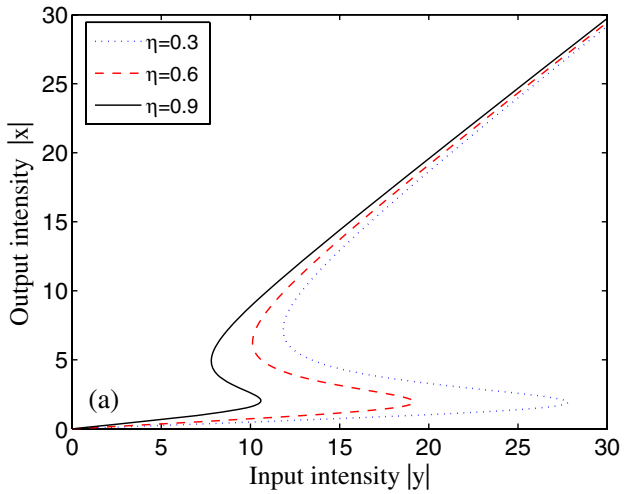

Fig. 3. (Color online) Output intensity $|x|$ versus input intensity $|y|$ for (a) open $\left(J_{2}=3 J_{1}\right.$, and $r_{0}=0.6 \gamma$ ), and (b) closed system $J_{1}=J_{2}=r_{0}=0$ ), with different strength of SGC effect $\eta$. Other parameters used are $\gamma_{31}=\gamma_{32}=30 \gamma_{21}=\gamma=3 \mathrm{MHz}, G_{c}=0.3 \gamma, \Omega_{d}=4 \gamma$, $\Lambda=0.5 \gamma, \phi=\pi / 3$, and $C=50 \gamma$.

$$
\begin{gathered}
E_{p}(L)=E_{p}^{T} / \sqrt{T}, \\
E_{p}(0)=\sqrt{T} E_{p}^{I}+R E_{p}(L),
\end{gathered}
$$

where $L$ is the length of the sample. The second term in the right-hand side of Eq. (17) describes the feedback from the mirror, which is essential for the generation of bistability. We note that no bistability occurs if $R=0$.

In the mean-field limit [30], using the boundary conditions in Eqs. $(\underline{16}, 17)$ and normalizing the field by letting $x=\frac{\mu_{13} E_{p}^{T}}{\hbar \sqrt{T}}$ and $y=\frac{\mu_{13} E_{p}^{I}}{\hbar \sqrt{T}}$, we can have the input-output relationship for the fields

$$
y=x-i C \rho_{31},
$$

where $C=\frac{N \omega_{p} L \mu_{13}^{2}}{2 c \varepsilon_{0} \hbar T}$ is the electronic cooperation parameter. The second term in the right-hand side of Eq. (18) is very important for $\mathrm{OB}$ to occur. The steady-state solution $\rho_{31}$ can be found by the set of equations in Eqs. (8)-(14), which is a set of coupled $9 \times 9$ algebraic equations after splitting them into real and imaginary parts. To give a clear illustration, we choose $\Delta_{p}=\Delta_{c}=0$ and $\gamma_{31}=\gamma_{32}=30 \gamma_{21}=\gamma$. All the parameters used are also set in units of constant $\gamma$ in the following numerical calculations.

\section{NUMERICAL RESULTS AND PHYSICAL ANALYSIS}

In this section, in Figures $3-7$, we present a few numerical results for the steady state solution of output field, in terms of field intensities for different corresponding parameters. First of all, we analyze how the strength of SGC modifies the bistable behavior, with all other parameters fixed. Figure 3 demonstrates the dependence of OB on the strength of SGC $\bar{\eta}$, with a comparison for open and closed systems. From Fig. 3, one can find that the threshold intensity of $\mathrm{OB}$ can be reduced both for open and closed systems, as the strength of SGC goes from $\eta=0.3$ to $\eta=1$. In this case, a smaller threshold intensity comes from the reduction in effective saturation intensity since SGC suppresses the radiative decay rate from state $|3\rangle$ to state $|1\rangle[31]$. As a result, we can have a desired bistable curve with a proper strength of SGC. Figure 3(b) also shows that the OB behavior in the closed system is similar to that in an open

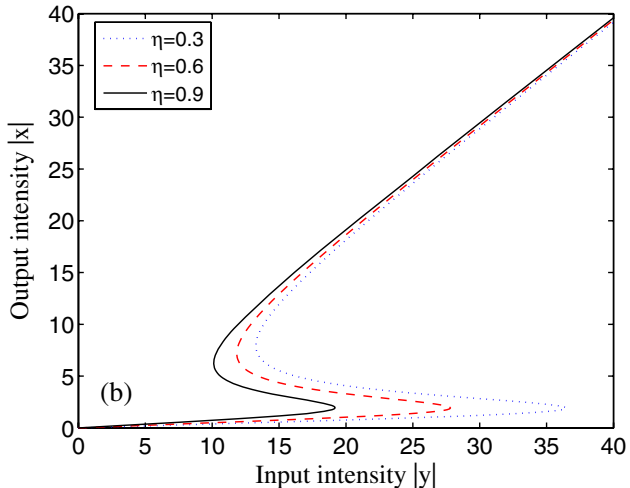



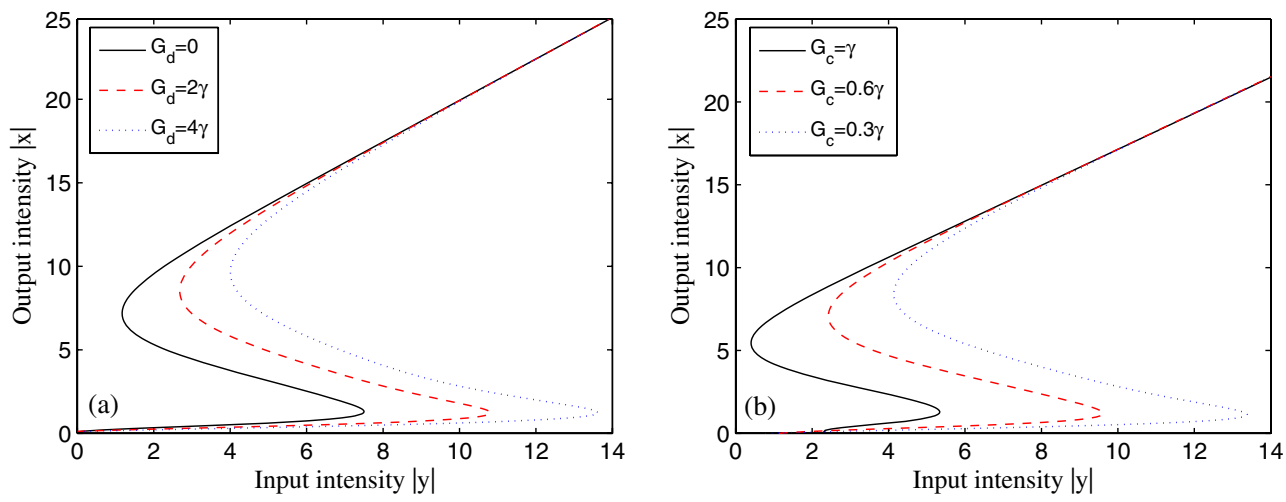

Fig. 4. (Color online) In the open atomic system, output intensity $|x|$ versus input intensity $|y|$, (a) for different amplitudes of microwave-field $G_{d}$ with $G_{c}=0.3 \gamma$ and (b) for different amplitudes of control field $G_{c}$ with $G_{d}=4 \gamma$. Other parameters used are the same as those in Fig. 3(a) except $\eta=1$.

system, but the threshold in closed system is obviously larger than that in the corresponding open system. A smaller threshold intensity is obviously favorable from viewpoint of experiments.

In Fig. 4, we analyze the effect of intensities of microwave and control fields on modifying the bistable behavior in the open atomic system. One can see that $\mathrm{OB}$ curve has a very sensitive dependence on the intensity of the driving fields $G_{j}(j=d, c)$. Moreover, the bistable threshold intensity increases as the intensity of microwave field increases or the intensity of control field decreases; meanwhile, the area in the corresponding hysteresis cycle becomes narrow for both cases. The physical interpretation of these results is clear. Either by increasing the intensity of control field or by decreasing the intensity of microwave field, the absorption for probe field on the transition $|1\rangle \leftrightarrow|3\rangle$ can be reduced dramatically, which makes the cavity field easier to reach saturation [18,28].

Figure 5 plots the curves of $\mathrm{OB}$ behavior with different values of the cooperation parameter $C$ and incoherent pumping rate. It is easy to see from Fig. 5(a) that the threshold of OB reduces dramatically when the cooperation parameter $C$ becomes smaller. For this reduction, the physical reason can be qualitatively explained as follows. From the term $C=N \omega_{p} L \mu_{13}^{2} / 2 c \varepsilon_{0} \hbar T$, one can find that the cooperation parameter $C$ is directly proportional to the electron number density $N$. Thus, the probe absorption increases as the electron density increases, which leads to the raise of a threshold intensity. Figure 5(b) shows that the threshold of OB also increases dramatically when the incoherent pumping rate increases, due to the quantum coherence [28]. In this $\Lambda$-type three-levels atomic system, the absorption of probe field for the transitions $|1\rangle$ to $|3\rangle$ increases with the incoherent pumping field driving between the levels $|1\rangle$ and $|3\rangle$. In this scenario, it is difficult to reach saturation for the probe field, and the bistable threshold intensity increases correspondingly.

Now, we investigate atomic exit rate, $r_{0}$, and atomic injection rates, $J_{1}$ and $J_{2}$, on bistable property in the open system. The curves of output intensity $|x|$ versus input intensity $|y|$ with different values of the atomic exit rate $r_{0}$ are plotted in Fig. 6(a), where we keep the atomic injection rate $J_{2}$ of level $|2\rangle$ as a constant. We find that the bistability threshold intensity goes down when the atomic exit rate from the cavity $r_{0}$ becomes large. More interestingly, the threshold of $\mathrm{OB}$ can also be controlled by the relative phase $\phi$ between the probe and driving fields. The effects of the relative phase $\phi$ on the OB is illustrated in Fig. 6(b), from which one can readily find that the $\mathrm{OB}$ is indeed sensitive to the relative phase $\phi$. The threshold intensity and related area in the hysteresis cycle for $\mathrm{OB}$ decrease when the relative phase varies from 0 to $\pm 3 \pi / 2$. In order to find the physical reason of these phenomena, we plot the absorption of probe field versus atomic exit rate, and versus relative phase between the probe and driving fields in Fig. 7. From Fig. 7(a), one can see that, under resonant condition, the absorption of probe laser field on the
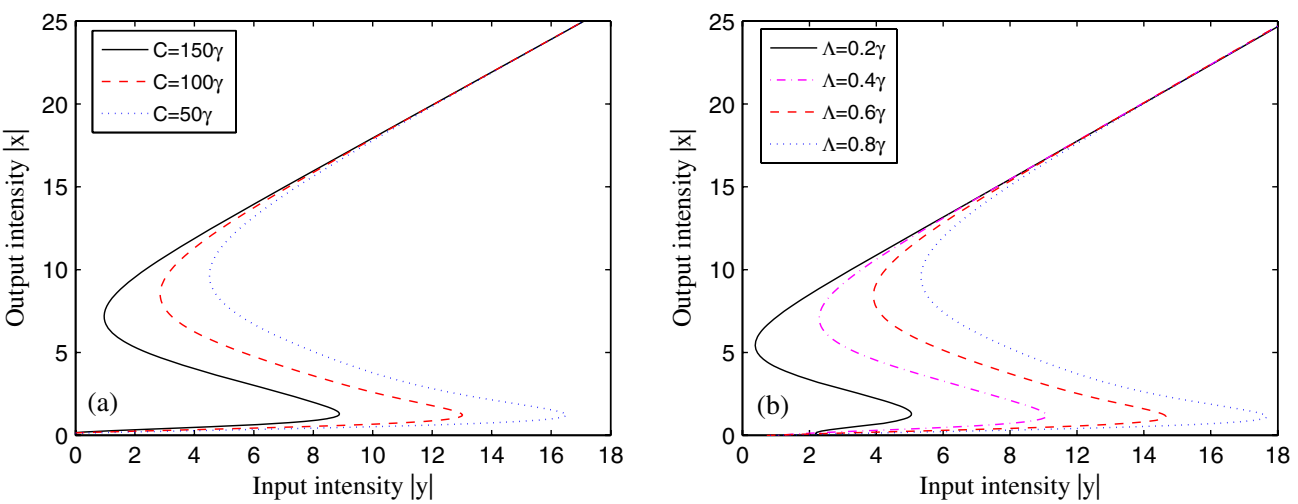

Fig. 5. (Color online) In the open atomic system, output intensity $|x|$ versus input intensity $|y|$, (a) for different values of cooperation parameter $C$ with $\Lambda=0.6 \gamma$ and (b) for different incoherent pumping rate $\Lambda$ with $C=100 \gamma$. Other parameters used are the same as those in Fig. $3(\mathrm{a})$ except $\eta=1$. 

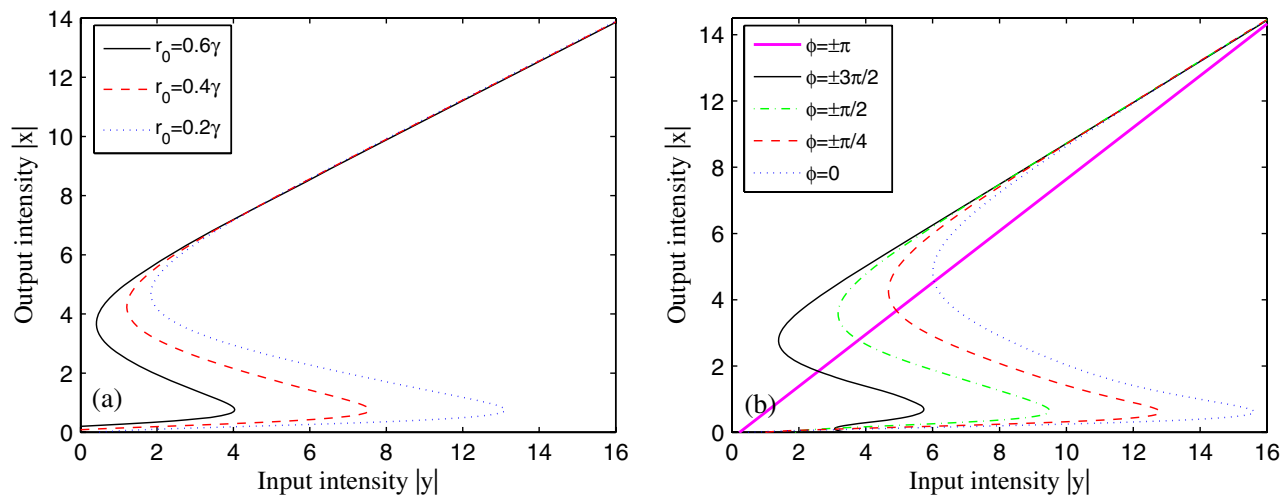

Fig. 6. (Color online) In the open atomic system, output intensity $|x|$ versus input intensity $|y|$ (a) for different atomic exit rate $r_{0}$ from the cavity with $\phi=\pi / 3$, (b) for different relative phase between probe and driving fields $\phi$ with $r_{0}=0.6 \gamma$. Other parameters used are the same as those in Fig. 3(a) except $\eta=1$.
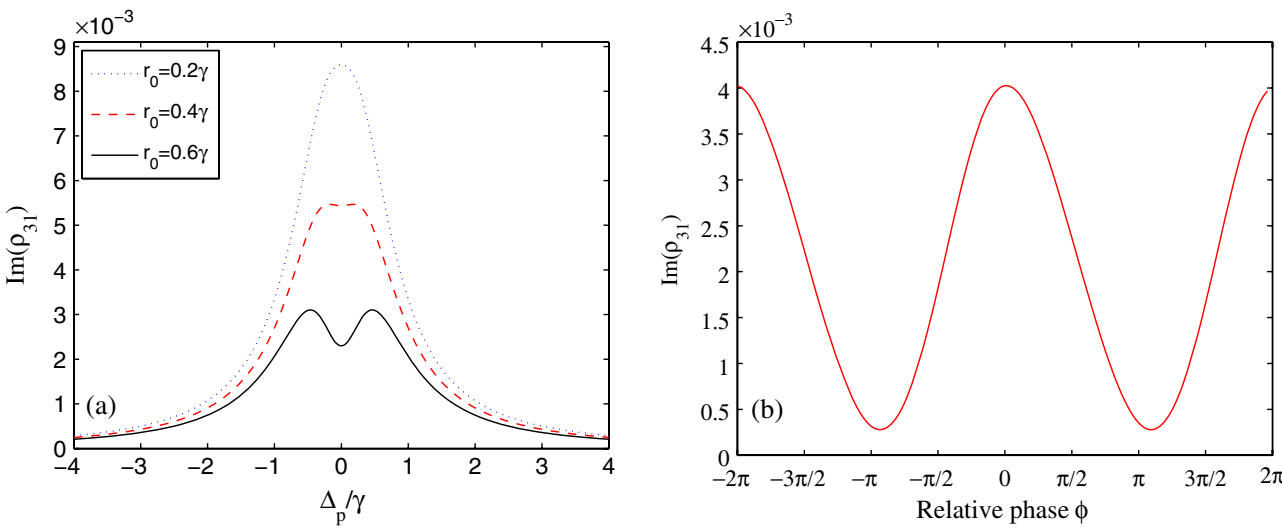

Fig. 7. (Color online) (a) Absorption of probe field $\operatorname{Im}\left(\rho_{31}\right)$ versus the detuning $\Delta_{p}$ for different atomic exit rates $r_{0}$ from the cavity with $\phi=\pi / 3$. (b) Absorption of probe field $\operatorname{Im}\left(\rho_{31}\right)$ versus the relative phase between probe and driving fields $\phi$ with $r_{0}=0.6 \gamma$. Other parameters used are the same as those in Fig. 3(a) except $\eta=1$.

transition $|3\rangle \leftrightarrow|1\rangle$ increases dramatically when the atomic exiting rate $r_{0}$ decreases. In other words, the decrease of atomic exit rate $r_{0}$ makes the cavity field more difficult to reach saturation. Figure 7(b) shows that the absorption of probe field increases gradually as we choose the relative phase $\phi$ as 0 , $\pi / 4, \pi / 2$, and $3 \pi / 2$. Therefore, the probe absorption is harder to reach saturation and the threshold intensity increases accordingly as shown in Fig. 6(a). In addition, it is found that an open atomic system turns to be a typical $\Lambda$-type three-level EIT medium when one chooses the relative phase $\phi= \pm \pi$. In this case, the probe absorption is closed to zero accordingly, as shown in Fig. 7(b), and the input field $E_{p}^{I}$ is approximately proportional to the transmitted field $E_{p}^{T}$. In other words, we can say that it is impossible for an OB to happen in this condition. Thus, the bistable behavior disappears in the case of $\phi= \pm \pi$, as illustrated by Fig. $\underline{6(b)}$.

\section{POSSIBLE REALIZATION IN EXPERIMENTS}

In this section, we would like to provide a possible realization of our proposed scheme in experiments. Similar to the experimental arrangement in [32], one can perform experiments with ${ }^{87} \mathrm{Rb}$ atomic vapors contained in a pyrex cell. The linearly polarized probe laser field excites the transition $5 S_{1 / 2}(F=1) \leftrightarrow 5 P_{1 / 2}(F=2)$, and the control laser and microwave field excite the transitions $5 S_{1 / 2}(F=2) \leftrightarrow 5 P_{1 / 2}(F=2)$

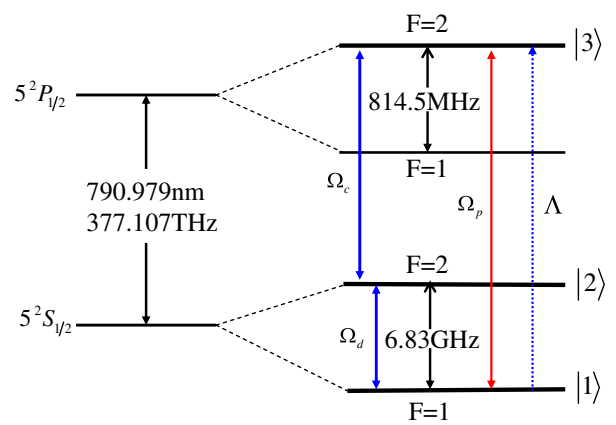

Fig. 8. (Color online) Hyperfine structure diagram and related fieldcoupling scheme for a three-level ${ }^{87} \mathrm{Rb}$ atom system.

and $5 S_{1 / 2}(F=1) \leftrightarrow 5 S_{1 / 2}(F=2)$, respectively, as illustrated in Fig. 8. An atomic beam is injected at the rates $J_{1}$ for level $|1\rangle$ and $\bar{J}_{2}$ for level $|2\rangle$, respectively. The atoms exit with a rate $r_{0}$ after interacting with the fields. We should note that all the values of parameters chosen in the present work are based on the experimental values in [33], such as the decay rates $\gamma_{i j}$ of ${ }^{87} \mathrm{Rb}$ atom, i.e., $\gamma_{31}=\gamma_{32}=3 \mathrm{MHz}$ and $\gamma_{21}=0.1 \mathrm{MHz}$.

\section{CONCLUSION}

In this work, we presented a model and related density matrix equations of motion for an open $\Lambda$-type configuration, where a 
microwave field drives a hyperfine transition between two lower states with SGC under electric dipole and rotating-wave approximations. With numerical results, we analyzed the optical bistability behavior by adjusting different parameters in the system. Under the steady-state condition, we find that the threshold intensity and related area in the corresponding hysteresis cycle of $\mathrm{OB}$ can be controlled by modulating the incoherent pumping rate, the cooperation parameter, SGC, and the intensity of driving fields. With the same parameters, we also provide a comparison between the open and closed systems. We find that the threshold intensity of $\mathrm{OB}$ in open system is less than the closed one. Furthermore, different from the closed system, the OB curves in an open system can also be adjusted by varying the atomic injection and exit rates. More interestingly, the appearance and disappearance of OB can be transformed mutually by adjusting the relative phase of three coherent fields with the existence of a strong SGC.

\section{ACKNOWLEDGMENT}

We would like to thank Ying $\mathrm{Wu}$ for his helpful discussions. The research is supported in part by the National Natural Science Foundation of China under Grant Nos. 11074036 and 10874050 and by the National Fundamental Research Program of China Grant No. 2007CB936300.

\section{REFERENCES}

1. M. O. Scully, S. Y. Zhu, and A. Gavrielides, "Degenerate quantum-beat laser: Lasing without inversion and inversion without lasing," Phys. Rev. Lett. 62, 2813-2816 (1989).

2. H. Wang, D. J. Goorskey, and M. Xiao, "Bistability and instability of three-level atoms inside an optical cavity," Phys. Rev. A 65, 011801(R) (2001).

3. S. E. Harris, "Electromagnetically induced transparency," Phys. Today 50(7), 36-42 (1997).

4. Y. F. Zhu, A. I. Rubiera, and M. Xiao, "Inversionless lasing and photon statistics in a V-type atomic system," Phys. Rev. A 53, 1065-1071 (1996).

5. Y. P. Niu and S. Q. Gong, "Enhancing Kerr nonlinearity via spontaneously generated coherence," Phys. Rev. A 73, 053811 (2006).

6. Y. Wuand L. Deng, "Achieving multi-frequency mode entanglement with ultra-slow multi-wave mixing," Opt. Lett. 29, 1144 (2004).

7. Y. Wu and X. Yang, "Highly efficient four-wave mixing in a double-Lambda system in an ultra-slow propagation regime," Phys. Rev. A 70, 053818 (2004).

8. Y. Zhang, U. Khadka, B. Anderson, and M. Xiao, "Temporal and spatial interference between four-wave mixing and six-wave mixing channels," Phys. Rev. Lett. 102, 013601 (2009).

9. Y. Wu and L. Deng, "Ultra slow optical solitons in a cold fourstate medium," Phys. Rev. Lett. 93, 143904 (2004).

10. Y. Wu and L. Deng, "Ultra slow bright and dark optical solitons in a cold three-state medium," Opt. Lett. 29, 2064 (2004).

11. Y. Wu, "Two-color ultraslow optical solitons via four-wave mixing in cold atom media," Phys. Rev. A 71, 053820 (2005).
12. W. X. Yang, A. X. Chen, L. Si, K. Jiang, X. Yang, and R. K. Lee, "Three coupled ultraslow temporal solitons in a five-level tripod atomic system," Phys. Rev. A 81, 023814 (2010).

13. L. Si, W. X. Yang, J. B. Liu, J. Li, and X. Yang, "Slow vector optics solitons in a cold five-level hyper V-type atomic system," Opt. Express 17, 7771 (2009).

14. S. Q. Gong, S. D. Du, Z. Z. Xu, and S. H. Pan, "Optical bistability via a phase fluctuation effect of the control field," Phys. Lett. A 222, 237-240 (1996).

15. A. Joshi and M. Xiao, "Optical multistability in three-level atoms inside an optical ring cavity,” Phys. Rev. Lett. 91, 143904 (2003).

16. A. Joshi, A. Brown, H. Wang, and M. Xiao, "Controlling optical bistability in a three-level atomic system," Phys. Rev. A 67, 041801(R) (2003).

17. J. H. Li, X. Y. Lu, J. M. Luo, and Q. J. Huang, "Optical bistability and multistability via atomic coherence in an N-type atomic medium," Phys. Rev. A 74, 035801 (2006).

18. J. Li, "Coherent control of optical bistability in a microwavedriven V-type atomic system," Physica D 228, 148 (2007).

19. X. Y. Lu, J. H. Li, J. B. Liu, and J. M. Luo, "Optical bistability via quantum interference in a four-level atomic medium," J. Phys. B 39, 5161 (2006).

20. J. Wu, X. Y. Lü, and L. L. Zheng, "Controllable optical bistability and multistability in a double two-level atomic system," J. Phys. B 43, 161003 (2010).

21. Z. P. Wang, "Optical bistability via coherent and incoherent fields in an $\mathrm{Er}^{3+}$-doped yttrium-aluminum-garnet crystal," Opt. Commun. 283, 3291-3295 (2010).

22. H. M. Gibbs, S. L. McCall, and T. N. C. Venkatesan, "Differential gain and bistability using a sodium-filled Fabry-Perot interferometer," Phys. Rev. Lett. 36, 1135-1138 (1976).

23. S. Singh, J. Rai, C. M. Bowden, and A. Postan, "Intrinsic optical bistability with squeezed vacuum," Phys. Rev. A 45, 5160-5165 (1992).

24. P. Galatola, L. A. Lugiato, M. G. Porreca, and P. Tombesi, "Optical switching by variation of the squeezing phase," Opt. Commun. 81, 175-178 (1991).

25. Z. Chen, C. Du, S. Gong, and Z. Z. Xu, "Optical bistability via squeezed vacuum input,” Phys. Lett. A 259, 15-19 (1999).

26. C. Liu, S. Gong, X. Fan, and Z. Xu, "Phase control of spontaneously generated coherence induced bistability," Opt. Commun. 239, 383-388 (2004).

27. M. O. Scully and M. S. Zubairy, Quantum Optics (Cambridge University, 1997), p. 161.

28. Y. Wu and X. Yang, "Electromagnetically induced transparency in $\mathrm{V}-, \lambda$-, and cascade-type schemes beyond steady-state analysis," Phys. Rev. A 71, 053806 (2005).

29. X. Fan, N. Cui, S. Tan, H. Ma, S. Gong, and Z. Xu, "Phase control of gain and dispersion in an open lambda-type inversionless lasing system,” J. Mod. Opt. 52, 2759-2769 (2005).

30. R. Bonifacio and L. A. Lugiato, "Optical bistability and cooperative effects in resonance fluorescence," Phys. Rev. A 18 1129-1144 (1978).

31. A. Joshi, W. Yang, and M. Xiao, "Effect of quantum interference on optical bistability in the three-level V-type atomic system," Phys. Rev. A 68, 015806 (2003).

32. A. Joshi, W. Yang, and M. Xiao, "Hysteresis loop with controllable shape and direction in an optical ring cavity," Phys Rev. A 70, 041802(R) (2004).

33. Y. Li and M. Xiao, "Electromagnetically induced transparency in a three-level $\Lambda$-type system in rubidium atoms,” Phys. Rev. A 51, R2703-R2706 (1995). 